\title{
Extract High Resolution 3D Quantitative Elemental Map Using a Combined HAADF-STEM and EDS Tomography
}

Yu Yuan ${ }^{1}$, Nicolas Brodusch ${ }^{1}$, Frédéric Voisard ${ }^{1}$, Boris Nijikovsky ${ }^{2}$, Audrey Moores ${ }^{1}$ and Raynald Gauvin $^{1}$

${ }^{1}$ McGill University, Montreal, Quebec, Canada, ${ }^{2}$ Facility for Electron Microscopy Research, McGill University, Montreal, Quebec, Canada

The high-angle annular dark field-scanning transmission electron microscopy (HAADF-STEM) has been widely used for tomography to characterize three-dimensional structure and composition at the nanometer scale [1]. Since the two-dimensional (2D) projection images obtained from HAADF-STEM have the characteristics of high resolution, the reconstruction yields a high signal to noise ratio (SNR). However, it only shows the Z-contrast as an aggregate information of all elements present. Energy dispersive spectroscopy (EDS) - STEM tomography, on the contrary, contains element-specific information, but is with low SNR because of the low counts of X-rays.

Zhong et al. [2] have developed a novel HAADF-EDS bimodal tomographic (HEBT) reconstruction technique which both keeps the element-specific feature of elemental maps from EDS and preserves the high SNR of Z-contrast images. Our work, based on Zhong's algorithm, further extends to the quantitative calculation of chemical composition for materials with phases of alloy compounds.

The algorithm links HAADF-STEM and EDS-STEM signals using the response ratio factor which is calculated with the assumption that both signals are linearly related to the projection of density distribution [2]. The reconstruction is performed using simultaneous iterative reconstruction technique (SIRT) to minimize the projection errors of HAADF and EDS signals simultaneously. The estimated density distributions (one for each element) are the function of the weight fraction and scattering cross-section. The 3D distribution of the weight fraction for each element can then be calculated with knowledge of the scattering cross-section.

To test the performance of our model, we use the simulation data of a phantom object as input and compare the reconstructed image with the phantom object. The simulation data was obtained from MC X-ray, a Monte Carlo program to simulate the interaction of an electron beam with the solid sample [3].

A 2D Ag-Au nanoparticle was designed with a core-shell structure. It is homogeneous along the $\mathrm{Y}$ axis and heterogeneous along the $\mathrm{X}$ and $\mathrm{Z}$ axes. The weight fractions of $\mathrm{Ag}$ and $\mathrm{Au}$ are presented in Figure 1 a) and $b$ ) respectively. The plane $Z=0$ is the top surface of the object. The core is composed of $10 \mathrm{wt} \%$ $\mathrm{Ag}$ and $90 \mathrm{wt} \% \mathrm{Au}$, while the shell is composed of $90 \mathrm{wt} \% \mathrm{Ag}$ and $10 \mathrm{wt} \% \mathrm{Au}$. Both HAADF and EDS signals are simulated for 16 different tilt angles ranging from -75 to 75 degrees with an interval of 10 degrees and 49 beam positions (along the $X$ axis) ranging from 0 to $144 \mathrm{~nm}$ with an interval of $3 \mathrm{~nm}$. The beam energy is $120 \mathrm{keV}$, and simulated electron number is 100,000. The X-ray signals for the Ag L $\beta$ line and $\mathrm{Au} \mathrm{M \alpha}$ line are used for the reconstruction. To simulate the real experiment condition, we use low acquisition time in the Monte Carlo simulation and added Poisson noise to the X-ray signals. The simulated X-ray counts in total are at the level of 20 and 50 for the Ag L $\beta$ line and Au M $\alpha$ line respectively. Figure 2 a) and b) present the reconstructed weight fraction distributions for Ag and Au with a pixel size $2.5 \times 2.5 \mathrm{~nm}$ and an iteration time of 100 . The core-shell structure is clearly observed, and the weight fraction values are close to the reference value for most of the pixels. However, for the top and bottom 
area of the shell where the shell is relatively thin, the boundary between the core and shell is not successfully recognized. It might be because the step sizes of both tilt angles and beam positions for the simulation data are too large.

The details of the algorithm and more examples of reconstruction for both simulated and experimental data will be presented.
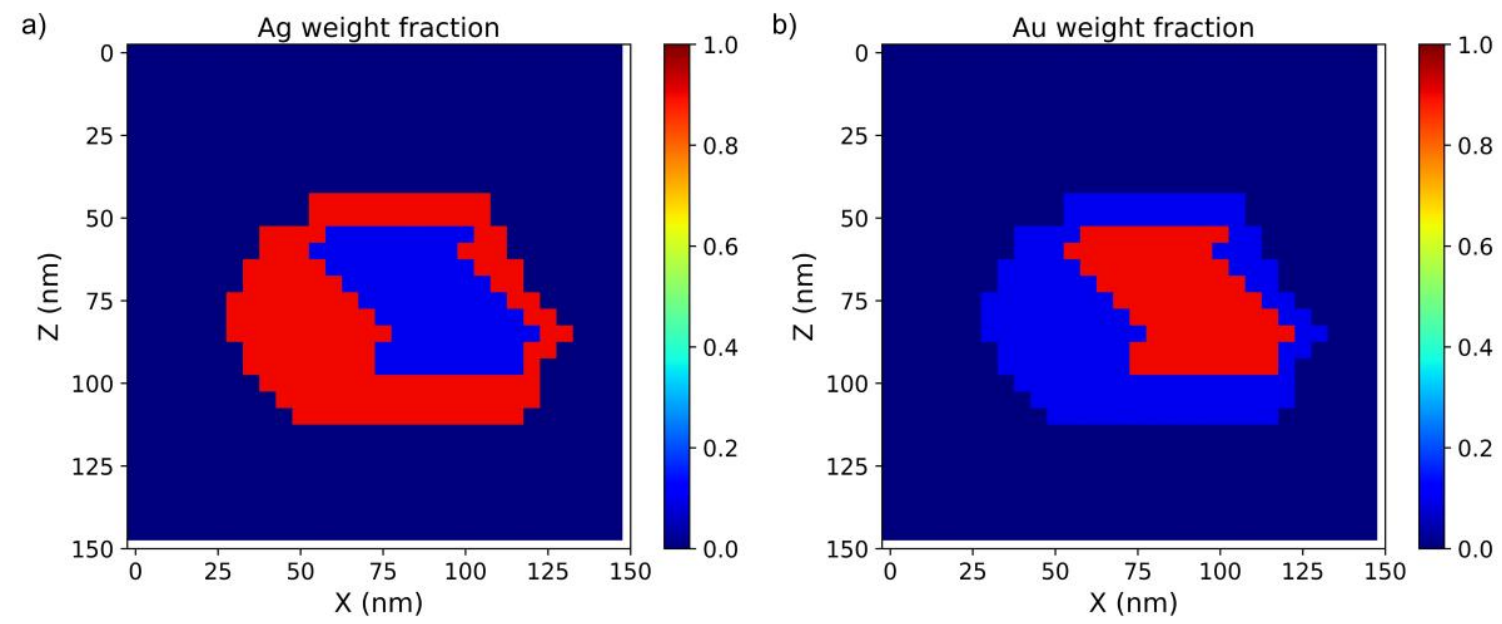

Figure 1. Element distribution on the $X-Z$ plane for the phantom object of a Ag-Au nanoparticle: weight fractions of a) $\mathrm{Ag}$ and b) $\mathrm{Au}$.
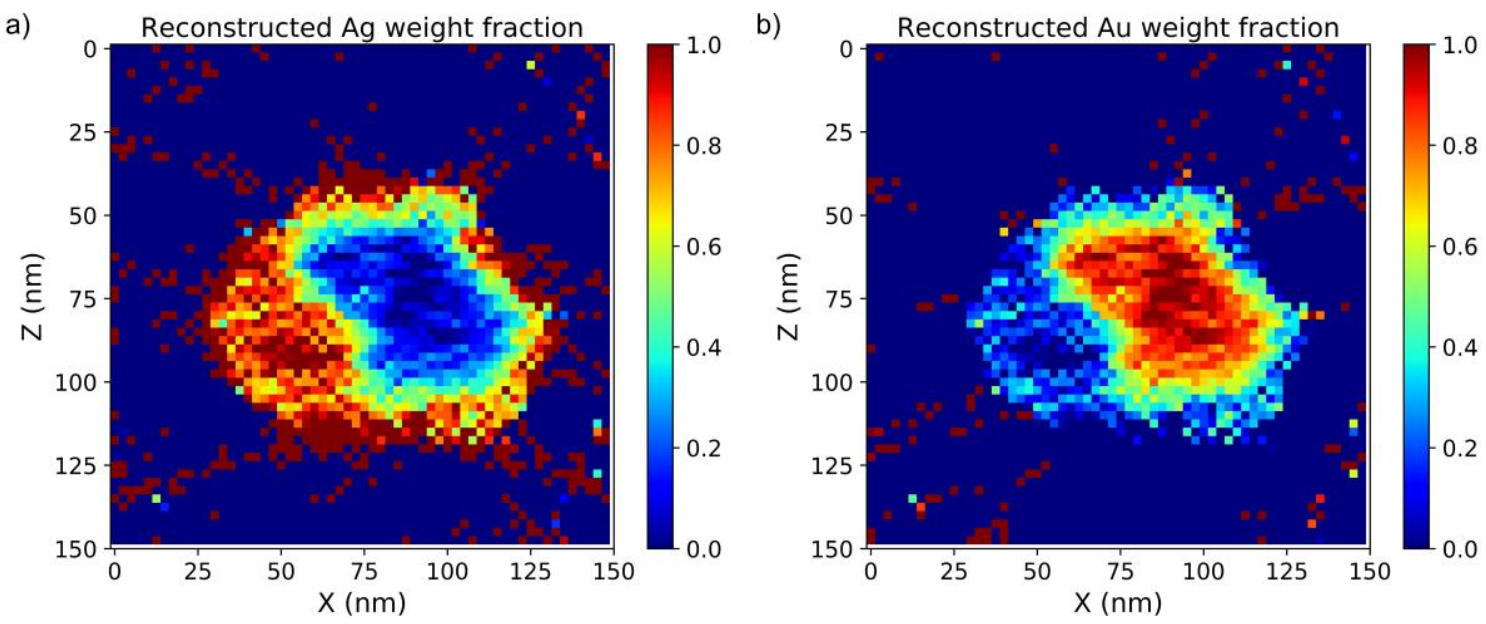

Figure 2. Reconstructed element distribution for the phantom object as sown in Figure 1: weight fractions of a) Ag and b) Au.

\section{References}

[1] S. J. Pennycook and P. D. Nellist. Scanning transmission electron microscopy: imaging and analysis, Springer Science \& Business Media (2011).

[2] Z Zhong, et al., Ultramicroscopy 174 (2017), p. 35-45.

[3] R Gauvin and P Michaud, Microscopy and Microanalysis 15 (2009), p. 488-489. 